\title{
Physiological effects of High-Flow Nasal Cannula and helmet CPAP in pediatric acute bronchiolitis
}

\author{
Giovanna Chidini ${ }^{1}$, Giorgio Conti ${ }^{2}$, Tommaso Mauri ${ }^{3}$, Stefano Scalia Catenacci ${ }^{3}$, Tiziana \\ Marchesi $^{3}$, Giada Dona ${ }^{3}$, Maria Adele Figini ${ }^{3}$, Giovanni Babini ${ }^{3}$, and Edoardo Calderini ${ }^{4}$ \\ ${ }^{1}$ Fondazione Fondazione IRCCS Ca' Granda Ospedale Maggiore Policlinico \\ ${ }^{2}$ Universita Cattolica del Sacro Cuore Facolta di Medicina e Chirurgia \\ ${ }^{3}$ Fondazione IRCCS Ca' Granda Ospedale Maggiore Policlinico \\ ${ }^{4}$ Paediatric Intensive Care Unit
}

March 2, 2022

\begin{abstract}
Objectives. Viral bronchiolitis represents one of the main cause of hospitalization for children in developed and incoming countries. Nasal High Flow (NHF) oxygen therapy improves oxygenation and reduces respiratory drive by enhancing carbon dioxide wash-out. However, little is known about physiological effects of noninvasive helmet-Continuous Positive Airway Pressure (h-CPAP) and NHF on respiratory Work Of Breathing (WOB) in bronchiolitis. Objective of the study is to measure Esophageal Pressure Time Product*minute (PTPes*min-1) as a surrogate for WOB in acute bronchiolitis during NH at different flow rates in comparison with conventional h-CPAP. Pressure Rate Product, physiological parameters and gas exchange were considered as secondary end-points. Methods. This is a physiological randomized crossover-study comparing four 20-minute trials: oxygen therapy delivered by non-fitting mask, NHF2l/Kg, NHF3l/Kg, h-CPAP $7 \mathrm{cmH} 2 \mathrm{O}$. Results. Were enrolled ten children with bronchiolitis needing noninvasive respiratory support. PTPes*min-1, Respiratory Rate and Pressure Rate Product decreased progressively from h-CPAP compared to NHF3l/kg, NHF2l/kg and oxygen mask ( $<<0.01$ for all parameters). SpO2:FiO2 increased during h-CPAP versus NHF3l/ $\mathrm{kg}, \mathrm{NHF} 2 \mathrm{l} / \mathrm{kg}$ and oxygen mask $(\mathrm{p}<0.01)$. Transcutaneous carbon dioxide tension was affected by increasing flow rate showing a progressive reduction at NHF3l/kg, NHF2l/kg and during h-CPAP compared to oxygen mask $(\mathrm{p}<0.001)$. Conclusions. h-CPAP was associated with a reduction in WOB and with a better oxygenation compared to oxygen mask and NHF trials; 2) NHF trials improved oxygenation and reduced the carbon dioxide tension compared to oxygen mask 3) NHF3l/ $\mathrm{kg}$ does not offer advantages compared to NHF $2 \mathrm{l} / \mathrm{kg}$ in improving oxygenation and carbon dioxide wash-out.
\end{abstract}

Giovanna Chidini ORCID 0000-0002-3807-1184

Physiological effects of High-Flow Nasal Cannula and helmet CPAP in pediatric acute bronchiolitis

Hosted file

image1.wmf available at https://authorea.com/users/463070/articles/558285-physiologicaleffects-of-high-flow-nasal-cannula-and-helmet-cpap-in-pediatric-acute-bronchiolitis

\section{Hosted file}

image2.wmf available at https://authorea.com/users/463070/articles/558285-physiologicaleffects-of-high-flow-nasal-cannula-and-helmet-cpap-in-pediatric-acute-bronchiolitis

Corresponding Author :

Giovanna Chidini, M.D. Mail to: giovanna.chidini@policlinico.mi.it 
Pediatric Intensive Care Unit, Fondazione IRCCS Cà Granda Ospedale Maggiore Policlinico

Via Della Commenda 9, 20100 Milano, Italy

Phone number +39-2-55032242

Fax number: +39-2-55032817

Abbreviated Title: Noninvasive Ventilation in Acute Bronchiolitis

Abstract

Objectives. Viral bronchiolitis represents one of the main cause of hospitalization for children in developed and incoming countries. Nasal High Flow (NHF) oxygen therapy improves oxygenation and reduces respiratory drive by enhancing carbon dioxide wash-out. However, little is known about physiological effects of noninvasive helmet-Continuous Positive Airway Pressure (h-CPAP) and NHF on respiratory Work Of Breathing (WOB) in bronchiolitis. Objective of the study is to measure Esophageal Pressure Time Product*minute (PTPes*min ${ }^{-1}$ ) as a surrogate for WOB in acute bronchiolitis during NH at different flow rates in comparison with conventional h-CPAP. Pressure Rate Product, physiological parameters and gas exchange were considered as secondary end-points.

Methods. This is a physiological randomized crossover-study comparing four 20-minute trials: oxygen therapy delivered by non-fitting mask, NHF2l/Kg, NHF3l/Kg, h-CPAP $7 \mathrm{cmH}_{2} \mathrm{O}$.

Results. Were enrolled ten children with bronchiolitis needing noninvasive respiratory support. PTPes* $\min ^{-1}$, Respiratory Rate and Pressure Rate Product decreased progressively from h-CPAP compared to NHF3l $/ \mathrm{kg}$, NHF2l $/ \mathrm{kg}$ and oxygen mask $\left(p<0.01\right.$ for all parameters). $\mathrm{SpO}_{2}: \mathrm{FiO}_{2}$ increased during h-CPAP versus NHF3l/kg, NHF2l/kg and oxygen mask $(p<0.01)$.

Transcutaneous carbon dioxide tension was affected by increasing flow rate showing a progressive reduction at NHF3l/kg, NHF2l/kg and during h-CPAP compared to oxygen mask $(p<0.001)$.

Conclusions. h-CPAP was associated with a reduction in WOB and with a better oxygenation compared to oxygen mask and NHF trials; 2) NHF trials improved oxygenation and reduced the carbon dioxide tension compared to oxygen mask 3) NHF3l/kg does not offer advantages compared to NHF $2 \mathrm{l} / \mathrm{kg}$ in improving oxygenation and carbon dioxide wash-out.

\section{INTRODUCTION}

Acute Respiratory Failure due to viral bronchiolitis represents one of the main cause of hospitalization for children both in developed and low income countries, with a Pediatric Intensive Care Units (PICUs) admission rate ranging from $2 \%$ to $6 \%^{1,2}$. In children, bronchiolitis is characterized by an increase in airway resistance due to small airways obstruction triggered by inflammation, a decrease in lung compliance due to alveolar collapse and atelectasis: all these leading to a rapid shallow breathing pattern ${ }^{3,4}$. In this setting, the application of 6-7 $\mathrm{cmH}_{2} \mathrm{O}$ Continuous Positive Airway Pressure results in alveolar recruitment, reduction in ventilation perfusion mismatch and airway stenting, thus leading to unloading of respiratory muscles, reduction in respiratory distress and improvement in gas exchange ${ }^{5-7}$. More recently, oxygen therapy delivered by Nasal High Flow (NHF) was introduced in adults and pediatric population ${ }^{8-13}$. Recent pediatric studies reported that NHF improves oxygenation, reduces the respiratory drive and prevent reintubation ${ }^{12,13}$. NHF therapy could result in several clinical benefits by enhancing carbon dioxide wash-out from upper airways and creating a low positive airways pressure. These effects, combined with an optimal airways humidification decreases the respiratory work of breathing and improves gas exchange in adults and children ${ }^{14-19}$.

However, little is known about the physiological effects of noninvasive helmet Continuous Positive Airway Pressure (h-CPAP) and NHF on pediatric respiratory work of breathing during the early phase of bronchiolitis. On one hand, NHF might be superior due to higher comfort and improved carbon dioxide clearance, on the other, CPAP delivers more constant and higher pressure, which may be critical for airway reopening and improving mechanics. To address the question on the more efficient support to reduce the work of breathing 
in children with severe acute bronchiolitis, we designed a physiological randomized crossover trial aimed at measuring the effects of four non-invasive respiratory supports: oxygen therapy delivered by oxygen Venturi mask, NHF at increasing flow rates $\left(2\right.$ and $3 \mathrm{l} / \mathrm{kg}$ ) and helmet $\mathrm{CPAP} 7 \mathrm{cmH}_{2} \mathrm{O}(\mathrm{h}-\mathrm{CPAP})$.

\section{MATERIALS AND METHODS}

The study was approved by the local institutional Ethics Committee and written informed consent was obtained from parents or legal guardian. The study was registered under ClinicalTrial.gov (NCT03689686). Consolidated Standards of Reporting Trials guidelines were followed, and the study was performed according to the ethical Declaration Standard laid down in the 1964 Helsinki as revised in 2008 ${ }^{20-21}$.

Study Design

This is a physiological crossover study comparing four 20-minute steps delivered in a computer generated random order: 1) Oxygen therapy delivered by non-fitting oxygen mask, equipped with Venturi system; 2) $\mathrm{NHF} 2 \mathrm{l} / \mathrm{Kg} ; 3) \mathrm{NHF} 3 \mathrm{l} / \mathrm{Kg} 4) \mathrm{h}-\mathrm{CPAP} 7 \mathrm{cmH}_{2} \mathrm{O}$ delivered through pediatric helmet. Concealed randomization was conducted centrally through a computer generated block-randomization schedule. A phone-call service was available h $24 / 7$ for patients' assignments to related group.

End Points

The primary end-point of the study was the difference in the Esophageal Pressure Time Product per minute $\left(\mathrm{PTP}_{\mathrm{es}} \mathrm{min}^{-1}\right)$, considered as a surrogate index for respiratory Work Of Breathing (WOB).

Per breath PTPes, the esophageal pressure swings during inspiration per breath $\left(\Delta \mathrm{Pes}_{\mathrm{Br}}\right)$, Pressure Rate Product (PRP, i.e. $\Delta \mathrm{Pes}_{\mathrm{Br}} *$ Respiratory Rate) and baseline respiratory variables were also measured. Modified Woods Clinical Asthma Score (M-WCAS) and EDIN Score (Echelle de Douleur et d'Inconfort du Nouveau Nè) were collected at the end of each study trials. Modified Woods Clinical Asthma Score (M-WCAS) is a combined score (0-10) used to evaluate the degree of respiratory distress in children with bronchiolitis. M-WCAS has been validated for use in hospitalized children with bronchiolitis and includes evaluation of oxygen saturation, inspiratory breath sounds, expiratory wheezing, use of accessory respiratory muscles, and cerebral function ${ }^{22}$. The EDIN scale uses five behavioral indicators of prolonged pain/discomfort: facial activity, body movements, quality of sleep, quality of contact with nurses, and consolability, with high interrater agreement and internal consistency ${ }^{23}$.

\section{Study Population}

All consecutive children ageing 1 month-2 years admitted to the PICU of the Fondazione Cà Granda, Ospedale Maggiore Policlinico (Milan, Italy) between October 2017 to April 2018 were screened to be eligible for the study. Inclusion criteria were: Age: $>30$ days and $<2$ years; $\mathrm{S} / \mathrm{F}<264$ while receiving additional oxygen as per clinical decision plus two of the followings: Respiratory Rate $(\mathrm{RR})>2 \mathrm{SD}$ according to age; active contraction of respiratory muscles; -paradoxical abdominal motion; - viral infection diagnosis consistent with clinical features and nasopharyngeal swab confirmation ${ }^{24,25}$. Exclusion criteria were: emergency need for intubation; Glasgow Coma Scale $<12$; $\mathrm{pH}<7.25$; cough reflex impairment; upper-airway obstruction; facial/gastric surgery; recurrent apnoeas; hemodynamic instability (need for vasopressor or inotropes); evidence of pneumothorax on lung echo or chest $\mathrm{x}$ ray; contraindications to insert the esophageal catheter.

The following variables were collected on PICU admission: age, sex, weight, Pediatric Index of Mortality 2 (PIM2), pediatric SOFA Score (pSOFA) ${ }^{26,27}$.

After enrollment and during the study in all patients ECG trace, transcutaneous gases, Respiratory Rate $(\mathrm{RR})$, Systolic and Diastolic blood pressure were monitored and displayed on a multiparametric PICU monitor (DraegerWerk AG and CO.KGaA, Luebeck, Germany).

Measurements and definitions.

A radio-opaque esophageal balloon 6 Fr (CareFusion, Linda, USA) was advanced through the nose for 15$20 \mathrm{~cm}$ to reach the stomach and inflated with the recommended volume of air $(0.3-0.5 \mathrm{ml})$. To ensure the 
correct position of the catheter, the esophageal balloon was positioned in the stomach to check the presence of positive deflection and then retracted until it reached the lower third of the esophagus as reported by the appearance of cardiac artifacts and fixed ${ }^{15}$. All traces were sampled at $100 \mathrm{~Hz}$, recorded for 5 mins at the end of each trial and offline analyzed on a dedicated data acquisition system (ICU Kleistek, Bari, Italy.) Esophageal pressure waveforms were analyzed offline by two senior physician not involved in the study and blinded respect the type of support and the name of the patient.

We measured the following variables:

1. Esophageal Pressure Time Product over a minute $\left(\mathrm{PTP}_{\mathrm{es}}{ }^{*} \mathrm{~min}^{-1}, \mathrm{cmH}_{2} \mathrm{O}{ }^{*} \mathrm{sec}^{*} \mathrm{~min}^{-1}\right)$ defined as the sum of the areas subtended by the esophageal inspiratory pressure curve over a period of 5 minutes analysis divided by the number of minutes; it represents a modification of the classic computation of the pressure-time product that requires measurement of the passive elastic recoil of the chest wall according to previous adult studies ${ }^{14-15}$

2. $\mathrm{PTP}_{\mathrm{es}} * \mathrm{~min}^{-1}$ divided by Respiratory Rate was PTPes per breath.

3. Esophageal pressure swings $\left(\Delta \mathrm{PesBr}, \mathrm{cmH}_{2} \mathrm{O}\right)$ during inspiration defined as the difference between end-inspiration and end-expiration esophageal pressure over a period of 5 minutes analysis (average value of 30 breaths recorded at the end of each step, after checking for artifacts) as a measurement of the patient's inspiratory effort according to previous adult studies ${ }^{14,15}$.

4. Pressure Rate Product (PRP, $\mathrm{cmH}_{2} \mathrm{O}^{*} \mathrm{~min}^{-1}$ ), defined as the mean $\Delta \mathrm{Pes}_{\mathrm{Br}}{ }^{*} \mathrm{RR}$ as a measurement of the patient's inspiratory effort over a minute, according to previous pediatric studies ${ }^{17-19}$.

Study protocol

Patients were kept in semirecumbent position. Sedation was kept constant during all the study with dexmedetomidine $0.5 \mathrm{mcg} / \mathrm{Kg} / \mathrm{hr}$ according to local PICU protocol ${ }^{28}$.

After a stabilization period with oxygen mask each patient received the four twenty mins ventilatory trials in randomized order (oxygen therapy with a non-fitting mask equipped with Venturi system to calculate $\mathrm{FiO}_{2}$, NHF $2 \mathrm{l} / \mathrm{Kg}$, NHF $3 \mathrm{l} / \mathrm{kg}$ and h-CPAP $7 \mathrm{cmH}_{2} \mathrm{O}$ ).

At end of trial 1, patients were switched over trial 2, 3 and finally to trial 4 . The first fifteeen minutes of each period were considered as a 'washout period' to minimize the carry-over effect. During washout periods, respiratory data were recorded but not considered for measurements. Physiological parameters were collected at the end of each trial to reduce the biases of treatment-time interaction effects on gas exchange.

$\mathrm{FiO}_{2}$ was set by the attending physician to target a peripheral saturation of 90-95\% during initial oxygen mask washout and kept constant during all phases.

In all patients, NHF was delivered through specific pediatrics nasal prongs (Fisher and Paykel Healthcare, Auckland, New Zealand). The set $\mathrm{FiO}_{2}$ during each phase was measured using a dedicated system (AIRVO2; Fisher and Paykel Healthcare) connected to nasal cannulas. h-CPAP was delivered by high fresh gas flow circuit with helmet with $7 \mathrm{cmH}_{2} \mathrm{O}$ CPAP level, according to previously published studies ${ }^{27,28}$. The attending physician evaluated treatment failure or success during the stabilization period and a pediatric intensive care senior consultant not involved in the study was always present for monitoring and treating potential adverse events. For safety reasons, the protocol included termination criteria, as follows: a) $\mathrm{SaO}_{2}<90 \%$ despite $\mathrm{FiO}_{2}>0.6$; b) transcutaneous carbon dioxide increment $>10 \%$ compared to baseline; c) need for emergency intubation d) haemodynamic instability defined as the need of vasopressors.

Sample size calculation and statistical analysis

The sample size was based on previous studies (14.15). We established the sample size (15 patients) to detect a difference at least of $20 \%$ in the primary end point, i.e. the PTPes* $\min ^{-1}$, with a Type 1 error of 0.05 and a desired power of $80 \%$ between NHF 2 l/Kg compared with baseline value on oxygen mask. Interim statistical analysis was pre-planned at 8-10-12 patients to detect excessive benefits from one treatment. The distribution data were determined using the Shapiro-Wilk analysis. Normally distributed variables are 
expressed as mean $\pm(\mathrm{SD})$ while median and interquartile range are used to report non-normally distributed variables. Differences between variables across trials were tested by parametric or non-parametric ANOVA (Friedman test) for repeated measures with post hoc Bonferroni comparison. Significance was taken as $p$ $<0.05$. Statistical analysis were performed with MedCalc for Windows.

\section{RESULTS}

The study was concluded at ten patients enrolled because interim statistical analysis showed that the primary end point was already reached with a high level of significance. Then, it was judged unethical to enroll other children requiring invasive maneuver such as the positioning of an esophageal balloon only for research purposes.

Study flowchart is depicted in Figure 1.

In our population, no children needed emergency intubation during the study period. All enrolled patients completed the study without interruptions. No major adverse events such as hemodynamic instability, bradycardia needing chest compression, cardiac arrest, or hypercapnic coma were reported during the study. One child was intubated 5 hours after the study because of persistent hypoxemia.

Baseline patients' characteristics are summarized in Table1 and Table 2.

Were enrolled ten children admitted to PICU because of viral bronchiolitis needing noninvasive respiratory support. Clinical suspicion of pulmonary infection (bacterial and/or viral) was defined as the presence of infiltrates visible on chest radiograph plus the presence at least 1 of 3 clinical variables: fever (body temperature $>38 \mathrm{C}^{\circ}$ ); leukocytosis/leukopenia and purulent secretions accompanied by abnormal breath sound. Nasopharyngeal and/or tracheal secretions were collected by use of a nonbronchoscopic blind technique at admission. Viral infection was detected by enzyme-linked immunoadsorbent assay ${ }^{24,25}$.

Causes of admission were: Respiratory Syncitial Virus infection (5 out of 10 patients, $50 \%$ ), Bocavirus (3 out of 10 patients, 30\%) and other viruses (Metapneumovirus and Rhinovirus (2 out of 10 patients, 20\%). The percentage of female gender was $50 \%$. The median age of our population was 11.5 [10-14.5] months, the median body weight was 10 [9-14] kg. The severity indexes at admission showed a median PIM2 score $2[1.8-2]$ and a median respiratory pSOFA score 2[2-2.7]. All children were enrolled early after admission and the study protocol started within a median time of three hours from PICU admission. Looking at PICU outcomes, no child failed the NRS trial. Enrolled children received a median period of NRS of 4[4.25-5] days with a median PICU LOS of 4[3-5]) days. No patient died during PICU and hospital stay. All patients survived at three and six months follow-up.

Patient's respiratory drive and effort parameters

Primary and secondary end point per protocol analysis are reported in Table 3. Figure 2 depicts the trend of primary end point and respiratory drive parameters over the study period.

PTPes over a minute decreased during h-CPAP (179 [97-376] $\left.\mathrm{cmH}_{2} \mathrm{O}^{*} \mathrm{sec}^{*} \mathrm{~min}^{-1}\right)$ compared to NHF 3l/kg (500[164-600] $\left.\mathrm{cmH}_{2} \mathrm{O}^{*} \mathrm{sec}^{*} \mathrm{~min}^{-1}\right), \mathrm{NHF} 2 \mathrm{l} / \mathrm{kg}\left(617[228-1100] \mathrm{cmH}_{2} \mathrm{O}^{*} \mathrm{sec}^{*} \mathrm{~min}^{-1}\right)$ and to oxygen mask (535[228$\left.701] \mathrm{cmH}_{2} \mathrm{O}^{*} \mathrm{sec}^{*} \mathrm{~min}^{-1}\right)(p<0.001)$.

$\triangle$ PESbr decreased during h-CPAP $\left(10[5-13] \mathrm{cmH}_{2} \mathrm{O}\right)$ compared to NHF3l/kg $\left(15[5.5-25] \mathrm{cmH}_{2} \mathrm{O}\right)$, HFNC $2 \mathrm{l} / \mathrm{kg}\left(17[4-26.5] \mathrm{cmH}_{2} \mathrm{O}\right)$ and to standard oxygen mask $\left(17[8.5-30.5] \mathrm{cmH}_{2} \mathrm{O}\right)(p<0.01)$.

Respiratory Rate decreased during h-CPAP (40 [35-45] breath $\left.\mathrm{min}^{-1}\right)$ compared to NHF3l/kg (45[4051]breath $\left.\mathrm{min}^{-1}\right)$, NHF2l/kg (45[37-55]breath $\left.\min ^{-1}\right)$ and to oxygen mask $\left(55[37-60]\right.$ breath $\left.\min ^{-1}\right)(p<0.01)$.

Pressure Rate Product (PRP) decreased during h-CPAP (410[207-61]) compared to NHF 3 l/kg (792 [241$1100])$ NHF $2 \mathrm{l} / \mathrm{kg}(600$ [171-1000]) and to oxygen mask (812[399-1500]) $(p<0.01)$.

Physiological Parameters and Comfort 
Physiological parameters across the study are reported in Table 3. Figure 3 depicts the physiological parameters at the different steps of the study. $\mathrm{FiO}_{2}$ was kept constant across the study and no variations $>5 \%$ were observed across the trials between $\mathrm{FIO}_{2}$ values displayed on AIRVO2 and values displayed by an external oximeter positioned on the inspiratory circuit limb.

$\mathrm{SpO}_{2}: \mathrm{FiO}_{2}$ showed a progressive significative increase during h-CPAP (326 [320-326]) versus NHF3l/kg (264[248-350]), NHF2l/kg (270[250-300]) and oxygen mask $(200[160-215])(p<0.01)$.

Transcutaneous carbon dioxide tension was decreased during NHF trials at $3 \mathrm{l} / \mathrm{kg}(33[31-45] \mathrm{mmHg}), 2 \mathrm{l} / \mathrm{kg}$ $(36[35-51] \mathrm{mmHg})$ and during h-CPAP $(33[31-42] \mathrm{mmHg})$ compared to oxygen mask $(46[3458] \mathrm{mmHg})(p$ $<0.001)$.

MWCAS score, an index of respiratory effort in acute bronchiolitis, showed a progressive decrease during h-CPAP (3[2.7-4]) compared to NHF 3l/kg (4.5[3-5]), to NHF 2l/kg (5[3-5]) and to standard oxygen mask $(6[5-6])(p<0.01)$. Heart Rate and mean arterial pressure were not affected by each trial.

Comfort improved and distress was decreased during the study with all tested type of NRS compared to oxygen mask (Table 3 ).

\section{DISCUSSION}

The major findings of this study was that increasing flow rate during NHF did not result in a reduction work of breathing compared to oxygen mask. Besides, both NHF trials were associated to a better oxygenation, a reduction in carbon dioxide tension values and in respiratory distress (m-WCAS score) compared to the oxygen mask without differences between 2 and 3l/kg. h-CPAP, instead, resulted in a significant reduction in all indexes of patient's respiratory effort and drive as well as oxygenation and $\mathrm{CO}_{2}$ levels compared both to NHF and oxygen mask trials.

Findings from our study were consistent with data reported by neonatal and pediatric studies conducted in children with acute viral bronchiolitis ${ }^{16-19}$.

However few papers are published comparing the physiological effects of NHF versus more conventional assistance such as standard oxygen therapy or noninvasive CPAP in pediatric patients.

In a multicenter, randomized, controlled trial, 1472 infants younger than 12 months with mild to moderate bronchiolitis were randomly assigned to receive either high-flow oxygen therapy [high-flow group] or standard oxygen therapy [standard-therapy group]. In this study the primary outcome was the escalation of care due to treatment failure defined by predetermined criteria [hypoxemia, tachypnea, worsening in respiratory distress].

In the above mentioned study the percentage of infants receiving escalation of care was $12 \%$ in the high-flow group, as compared with $23 \%$ in the standard-therapy group. No significant differences were observed in the duration of hospital stay or the duration of oxygen therapy. Authors concluded that infants with bronchiolitis receiving NHF outside a PICU, had significantly lower rates of escalation of care due to treatment failure than those in the group that received standard oxygen therapy ${ }^{31}$.

In a single center prospective physiological trial on 21 children with bronchiolitis, Weiler et al. determined the effects of three different flow rates $[0.5,1,1.5$ and $2 \mathrm{l} / \mathrm{Kg}]$ on pressure rate product. In this study the optimal flow rate to reduce effort of breathing in infants and young children was approximately 1.5-2.0 $\mathrm{L} / \mathrm{kg} /$ minute with more benefit seen in children [?] $8 \mathrm{~kg}^{19}$.

In a study comparing NHF2l/kg vs NHF3 l/kg including 244 infants with mild to moderate bronchiolitis, Milesi et al. reported a comparable failure rate between groups in the first 48 hours from admission. Worsening comfort was the more common cause of failure in $3 \mathrm{l} / \mathrm{kg}$ group without differences in intubation rate between groups ${ }^{32}$.

$\mathrm{H}$-CPAP was reported to be efficient in increasing end expiratory lung volume and compensating for airway pressure oscillations without carbon dioxide rebreathing at high fresh gas flow rates, with a more constant pressurization compared to other interfaces (nasal or oronasal mask). In infants with severe viral 
bronchiolitis, $6 \mathrm{cmH}_{2} \mathrm{O}$ h-CPAP reduced the PTPes and was associated with the greatest muscle unloading, improvement in breathing pattern, and favourable clinical outcome $\mathrm{e}^{5-7}$. In a prospective RCT conducted on children ageing 2-24 months with ARF, Vitaliti et al. compared h-CPAP and NHF2l/kg versus a control group of patients treated with oxygen therapy and standard medical treatment. In this study, both NHF2l/kg and h-CPAP were associated with an improvement in oxygenation and respiratory parameters compared with the control group. H-CPAP was associated with a more rapid improvement and resolution of respiratory impairment compared to NHF2l $/ \mathrm{kg}^{33}$. Similar data are reported in a multicenter RCT including 142 infants with viral bronchiolitis comparing $7 \mathrm{cmH}_{2} \mathrm{O}$ CPAP vs NHF $2 \mathrm{l} / \mathrm{kg}$. Data analysis suggested a relative risk of success higher with $\mathrm{h}-\mathrm{CPAP}^{34}$. In a recent metanalysis including 2121 children with mild to moderate bronchiolitis, NHF $2 \mathrm{l} / \mathrm{kg}$ decreased the rate of failure compared to standard oxygen therapy, but it was not associated with an improvement compared to conventional nasal CPAP treatment. An increased risk of failure in NHF group compared to nasal CPAP group was reported. However NHF was superior to standard oxygen treatment in reducing risk of failure and hospital length of stay ${ }^{35}$.

In an open phase-four RCT conducted on 202 children with severe bronchiolitis, NHF did not reduced time on oxygen compared to standard treatment, thus suggesting that NHF does not affect outcome variables in more severe disease ${ }^{36}$.

Data from our study confirm that different flow rates during NHF trials were not associated with a reduction in patient's effort parameters such as PTPes, DeltaPes ${ }_{\mathrm{Br}}, \mathrm{RR}$ and PRP, thus reinforcing, from a physiological point of view, the above mentioned clinical studies. NHF trials only resulted in a better oxygenation and a more efficient reduction in carbon dioxide tension. To explain this phenomenon we could hypothesize that, increasing the flow rate, the $\mathrm{CO}_{2}$ washout from the upper airways would be enhanced, mimicking a CPAP effect.

The main physiological h-CPAP effects in infants results from airway splinting, end-expiratory lung volume increase, reduced ventilation/perfusion mismatch, and respiratory pattern stabilization.

Our data confirm that h-CPAP significantly reduced all indexes of respiratory work of breathing and improved oxygenation compared to NHF trials and oxygen mask. We can hypothesize that this effect could be related to the more constant pressurization applied to the respiratory system with helmet interface compared to nasal prongs or cannulas, leading to a more efficient recruitment of collapsed alveolar units. Both h-CPAP and NHF trials decreased carbon dioxide tension compared to standard oxygen, but only h-CPAP associated improved carbon dioxide clearance with decreased respiratory effort. We might speculate that this effect of h-CPAP results both from improved ventilation/perfusion matching and to decreased carbon dioxide production due to lower work of breathing.

Our study has some limitations that need to be considered for an optimal interpretation of the results. First, this is a short-term physiological study including a small sample of children not including the investigation on possible long-term clinical consequences. Second, the study was not blinded for treatment, since it was impossible to hide the interface from caregivers. However, the analysis of the traces was conducted on a deidentified database (researchers were blinded respect to the name of the patient and the phases of the trial during the traces analysis). Third, carbon dioxide tension were collected with a transcutaneous gas analyzer and not from an arterial blood gas sample whereas oxygenation was determined by $\mathrm{SpO}_{2}: \mathrm{FiO}_{2}$ ratio according to previous pediatric studies ${ }^{37}$.

In conclusion, data from our study suggest that: 1) NHF 2 and 3l/kg could improve oxygenation and reduce the carbon dioxide tension compared to oxygen mask 2) NHF3l/ $\mathrm{kg}$ does not offer advantages compared to NHF2l/kg in improving oxygenation, carbon dioxide wash-out or respiratory effort parameters 3) h-CPAP was associated with a reduction in all indexes of work of breathing and with a better oxygenation compared both to oxygen mask and NHF trials 2 and $3 \mathrm{l} / \mathrm{kg}$.

Further clinical trials are needed to explore the possible long term effects of different NRS in pediatric acute respiratory failure. 


\section{ACKNOWLEDGEMENT/DISCLOSURE}

Funding: None

Conflict of interest: No potential conflict of interest was reported by the authors

Financial Disclosures: None

\section{REFERENCES}

1. Balfour Lynn RE, Marsh G, Gorayi D et al; Non-invasive ventilation for children with acute respiratory failure in the developing world: literature review and implementation example. Paediatric Respiratory Reviews. 2014; 15: 181-187.

2. Murthy S, Kissoon N. Management of severe viral iNHFections in the pediatric intensive care unit. Journal of Pediatric Intensive Care. 2014; 3: 205-216.

3. Ganu SS, Gautam A, Wilkins B et al; Increase in use of non-invasive ventilation for iNHFants with severe bronchiolitis is associated with decline in intubation rates over a decade. Intensive Care Medicine. 2012; 38: 1177-1183.

4. Essouri S, Laurent M, Chevret L et al; Improved clinical and economic outcomes in severe bronchiolitis with pre-emptive nCPAP ventilatory strategy. Intensive Care Medicine. 2014; 40: 84-91.

5. Cambonie G, Milési C, Jaber S et al; Nasal continuous positive airway pressure decreases respiratory muscles overload in young iNHFants with severe acute viral bronchiolitis. Intensive Care Medicine. 2008; 34: 1865-1872.

6. Milési C, Matecki S, Jaber $\mathrm{S}$ et al; $6 \mathrm{cmH}_{2} \mathrm{O}$ continuous positive airway pressure versus conventional oxygen therapy in severe viral bronchiolitis: a randomized trial. Pediatr Pulmonol. 2013; 48: 45-51.

7. Essouri S, Durand P, Chevret L et al; Optimal level of nasal continuous positive airway pressure in severe viral bronchiolitis. Intensive Care Medicine. 2011; 37: 2002-2007.

8. Papazian L, Corley A, Hess D, Fraser JF, Frat JP et al; Use of high-flow nasal cannula oxygenation in ICU adults: a narrative review. Intensive Care Medicine. 2016; 42: 1336-1349.

9. Nishimura M. High-flow nasal cannula oxygen therapy in adults: physiological benefits, indication, clinical benefits, and adverse effects. Respir Care. 2016; 61: 529-541.

10. Maggiore SM, Idone FA, Vaschetto R, Festa R et al; Nasal highflow versus Venturi mask oxygen therapy after extubation. Effects on oxygenation, comfort, and clinical outcome. Am J Respir Crit Care Med. 2014; 190: 282-288.

11. Frat JP, Thille AW, Mercat A, Girault C et al; High-flow oxygen through nasal cannula: FLORALI Study Group; REVA Network. New Eng J Med. 2015; 372: 2185-2196.

12. Lee JH, Rehder, KJ, Williford, L, Cheifetz, IM, Turner DA. Use of high flow nasal cannula in critically ill iNHFants, children, and adults: a critical review of the literature. Intensive Care Medicine. 2013; 39: 247-257.

13. Nielsen KR, Ellington EL, Gray AJ et al; Effect of High-Flow Nasal Cannula on Expiratory Pressure and Ventilation in INHFant, Pediatric, and Adult Models. Respiratory Care. 2017; DOI: 10.4187/respcare.05728.

14. Mauri T, Turrini C, Eronia N, Grasselli G et al; Physiologic effects of high-flow nasal cannula in acute hypoxemic respiratory failure. Am J Respir Crit Care Med. 2017; 195: 1207-1215.

15. Mauri T, Alban L, Turrini C, Cambiaghi B et al; Optimum support by high-flow nasal cannula in acute hypoxemic respiratory failure: effects of increasing flow rates. Intensive Care Medicine. 2017; $43: 1453$ $-1463$.

16. Shetty SM, Hickey A, Rafferty GF, Peacock JL et al; Work of breathing during CPAP and heated humidified high-flow nasal cannula. Arch Dis Child Fetal Neonatal. Ed 2016; 0: F1-F4.

17. Rubin S, Guman A, Deakers T, Khemani R, Ross P, Newth CJ. Effort of breathing in children receiving high flow nasal cannulas. Pediatr Critic Care Med. 2014; 15: 1-6.

18. Pham TM, O’Malley L, Mayfield S, Martin S, Schibler A . The effect of high flow nasal cannula therapy on the work of breathing in iNHFants with bronchiolitis. Pediatr Pulmonol. 2015; 50: 713 -720.

19. Weiler T, Kamerkar A, Hotz J, Ross PA, Newth CJ, Khemani RG. The relationship between High 
Flow Nasal Cannula Flow Rate and Effort of Breathing in Children. J Pediatr. 2017; 189: 66-71.

20. Begg C, Cho M, Eastwood S et al; Improving the quality of reporting of randomized controlled trials. The CONSORT statement. JAMA 1996; 276: 637-639.

21. Puri KS, Suresh KR, Gogtay NJ et al; Declaration of Helsinki, 2008: Implications for stakeholders in research. J Postgrad Med. 200; 55: 131-134.

22. Wood DW, Downes JJ, Lecks HI. A clinical scoring system for the diagnosis of respiratory failure. Preliminary report on childhood status asthmaticus. Am J Dis Child 1972; 123: 122.

23. Debillon T, Zupan V, Ravault N et al; Development and initial validation of the EDIN scale, a new tool for assessing prolonged pain in preterm iNHFants. Arch Dis Child Fetal Neonatal Ed. 2001; 85: F36-F41.

24. Mathers LH, Frankel LR. Stabilization of the critically ill child. In: Behrman RE, Kliegman RM, Jenson HB, eds. Nelson Textbook of Pediatrics, 17th ed Philadelphia, PA: Saunders; 2004:279-313.

25. Niederman MS, Mandell LA, Anzueto A et al; American Thoracic Society Guidelines for the management of adults with community acquired pneumonia: diagnosis, assessment of severity, antimicrobial therapy, and prevention. Am J Respir Crit Care Med. 2001; 16: 1730 -17.

26. Slater A, Shann F, Pearson G. Paediatric Index of Mortality (PIM) Study Group: PIM2: A revised version of the Paediatric Index of Mortality. Intensive Care Medicine. 2003; 29:278-285.

27. Matics TJ, Sanchez-Pinto LN. Adaptation and Validation of a Pediatric Sequential Organ Failure Assessment Score and Evaluation of the Sepsis-3 Definitions in Critically Ill Children. JAMA Pediatr. 2017; 171: e172352.

28. Venn RM, Hell J, Grounds RM. Respiratory effects of dexmedetomidine in the surgical patient requiring intensive care. Crit Care. 2000; 4: 302-308.

29. Chidini G, Piastra M, Marchesi T, De Luca D et al; Continuous positive airway pressure with helmet versus mask in iNHFants with bronchiolitis: an RCT. Pediatrics. 2015; doi: 10.1542/peds.2014-1142.

30. Chidini G, Calderini E, Pelosi P. Treatment of acute hypoxemic respiratory failure with continuous positive airway pressure delivered by a new pediatric helmet in comparison with a standard full face mask: a prospective pilot study. Pediatr Crit Care Med. 2010; 11: 502-508.

31. Franklin D, Babl FE, Schlapbach LJ, Oakley E et al; . A randomized trial of high-flow therapy in iNHFants with bronchiolitis. N Engl J Med. 2018; 378: 1121-1131.

32. Milesi C, Pierre AF, Deho A, Liets JM et al; Multicenter randomized controlled trial of a 3-L $/ \mathrm{kg} / \mathrm{min}$ versus $2-\mathrm{L} / \mathrm{kg} / \mathrm{min}$ high-flow nasal cannula flow rate in young iNHFants with severe viral bronchiolitis [TRAMONTANE 2]. Intensive Care Medicine. 2018; 44: 1870-1878.

33. Vitaliti G, Vitaliti MG, Finocchiaro MC, Di Stefano VA et al; Randomized Comparison of Helmet CPAP Versus High-Flow Nasal Cannula Oxygen in Pediatric Respiratory Distress. Respir Care. 2017; 62: 1036-1042.

34. Milesi C, Essouri S, Pouyau R, Liet J-M et al; High flow nasal cannula [HFNC] versus nasal continuous positive airway pressure [nCPAP] for the initial respiratory management of acute viral bronchiolitis in young iNHFants: a multicenter randomized controlled trial [TRAMONTANE study]. Intensive Care Medicine. 2017; 43: 209-216.

35. Lin J, Zhang Y, Xiong L, Liu S, Gong C, Dai J. High-flow nasal cannula therapy for children with bronchiolitis: a systematic review and meta-analysis. Arch Disease Child. 2019; 0: 1-13.

36. Kepreotes E, Whitehead B, Attia J, Oldmeadow C, Collison A, Searles A, et al. High-flow warm humidified oxygen versus standard low-flow nasal cannula oxygen for moderate bronchiolitis [HFWHO RCT]: an open, phase 4, randomised controlled trial. Lancet. 2017; 389: 930-939.

37. Mayordomo-Colunga J, Pons M, Lopez Y, et al; Predicting noninvasive ventilation failure in children from the SpO2/FiO2 (SF) ratio Intensive Care Medicine. 2013; 39: 1095-1103.

\section{Hosted file}

Legends.docx available at https://authorea.com/users/463070/articles/558285-physiologicaleffects-of-high-flow-nasal-cannula-and-helmet-cpap-in-pediatric-acute-bronchiolitis

\section{Hosted file}


Tables.docx available at https://authorea.com/users/463070/articles/558285-physiologicaleffects-of-high-flow-nasal-cannula-and-helmet-cpap-in-pediatric-acute-bronchiolitis
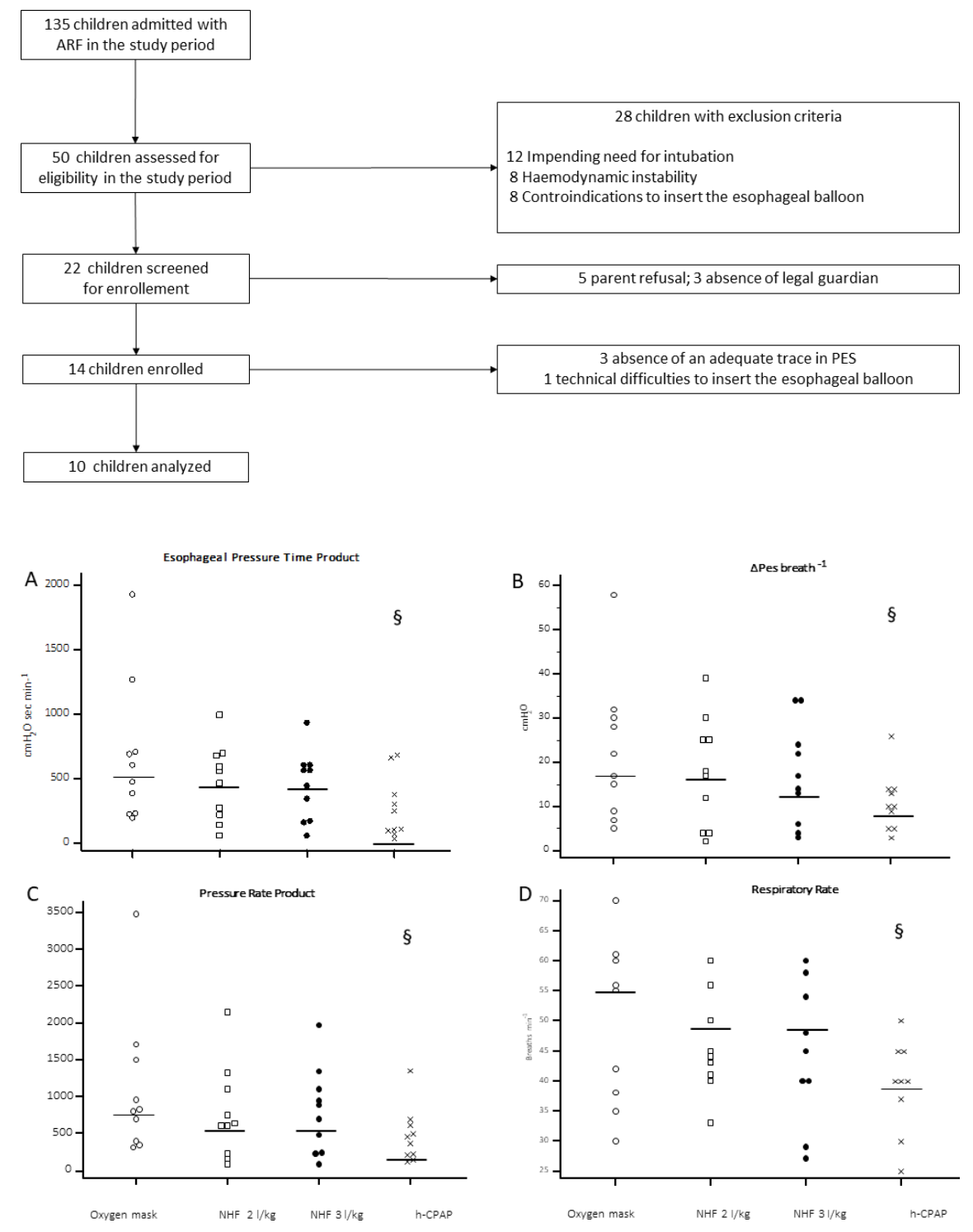

